www.nature.com/pj

\title{
Preparation of novel polyimide hybrid materials by multi-layered charge-transfer complex formation
}

\author{
Ryohei Watari ${ }^{1}$, Masamichi Nishihara ${ }^{2}$, Hiroo Tajiri ${ }^{3}$, Hideyuki Otsuka ${ }^{1,4}$ and Atsushi Takahara ${ }^{1,2,4}$ \\ A versatile method for the post-modification of sulfonated polyimides by charge-transfer (CT) complex formation between \\ electron-deficient naphthalenediimide units in polyimide and electron-rich dihydroxynaphthalene was developed. CT complex \\ hybrid films whose physicochemical properties, such as mechanical strength, differed from those of the original polyimide films \\ were successfully fabricated.
}

Polymer Journal (2013) 45, 839-844; doi:10.1038/pj.2012.222; published online 12 December 2012

Keywords: CT complex; molecular aggregation structure; physical properties; polyimide

\section{INTRODUCTION}

Polyimides are important for industrial materials in the electronics, ${ }^{1}$ photonics $^{2}$ and aerospace ${ }^{3}$ industries because of their excellent thermal stability, relatively low dielectric constants, high chemical resistance and good mechanical properties. ${ }^{4-6}$ These properties of polyimides mainly result from the molecular orientations of the polyimide main chains and the structural stability of the rigid aromatic groups in the main chains. ${ }^{7}$ Molecular interactions among the rigid aromatic groups also affect these properties. Intramolecular and intermolecular charge-transfer (CT) interactions, which are typical interactions, contribute to a polyimide's mechanical and thermal stabilities and color. ${ }^{7}$ Aromatic polyimides can form CT complexes with various donor compounds, ${ }^{8}$ and the physical properties of CT complex have been evaluated. 1,4,5,8naphthalenediimide (NDI) units have been used as one of the electron-deficient monomers for polyimides. NDI units have been used to prevent the hydrolysis of imide rings, ${ }^{9}$ and the obtained sulfonated polyimides (SPIs) have been used as polymer electrolyte membranes for polymer electrolyte fuel cells. ${ }^{10,11}$

Stiff and planar NDI derivatives have been used not only as repeating units for polyimides but also as building blocks for supramolecular structures, using CT complex formation with donor molecules such as dialkoxynaphthalene derivatives. ${ }^{12-18}$ Iverson et al. ${ }^{12,13}$ have reported folded structures formed by CT complex interactions between NDI oligomers and dialkoxynaphthalene oligomers. Matile and co-workers ${ }^{14,17}$ have formed rigid-rod $\beta$-barrels with NDI units and have controlled their structures by adding structurally optimal dialkoxynaphthalene derivatives. Iverson et al. ${ }^{19}$ have also applied the CT complex formation between NDI and dialkoxynaphthalene to the interactions between the side chains of polymers and have studied the macromolecular assembly of a donor polymer and an NDI-containing polymer. However, CT complex formation by NDI-containing polyimides, and an evaluation of the physical properties of these complexes, has not been reported.

In this paper, we report a CT complex formation approach to prepare polyimide hybrid materials by mixing an NDI-containing SPI and a low molecular weight donor molecule (Figure 1). Generally, the modification of polyimides is more limited than that of other polymers, such as vinyl polymers, because of their chemical stability and their poor solubility in common solvents as a result of their highly rigid structures. Because of these practical problems, the introduction of functionalities in polyimides is conducted by polymerizing pre-functionalized monomers. In this paper, we propose a simple and versatile method for the functionalization of polyimide films, without modifying the chemical structures of the original polyimides, by CT complex formation between NDI units and a dihydroxynaphthalene.

In addition, the formation of a multi-layer CT complex provides a strong binding constant compared with a 1:1 CT complex. ${ }^{13}$ Therefore, the CT hybrid material, which consists of a CT complex between NDI-containing SPI and a low molecular weight donor molecule, is hypothesized to show a larger binding constant than the intact NDI-containing SPI, as well as a higher mechanical strength. In this study, we compare various characteristics of CT hybrid materials and SPIs, including their visible spectra and their mechanical strength, and we discuss the effect of CT complex formation on the functionality of polymeric hybrid materials.

\footnotetext{
${ }^{1}$ Graduate School of Engineering, Kyushu University, Fukuoka, Japan; ${ }^{2}$ World Premier International Research Center Initiative, International Institute for Carbon-Neutral Energy Research (WPI-I2CNER), Kyushu University, Fukuoka, Japan; ${ }^{3}$ Japan Synchrotron Radiation Research Institute (JASRI/SPring-8), Hyogo, Japan and ${ }^{4}$ Institute for Materials Chemistry and Engineering, Kyushu University, Fukuoka, Japan

Correspondence: Professor A Takahara, Institute for Materials Chemistry and Engineering, Kyushu University, 744 Motooka, Nishi-ku, Fukuoka 819-0395, Japan.

E-mail: takahara@cstf.kyushu-u.ac.jp

Received 19 September 2012; revised 25 October 2012; accepted 28 October 2012; published online 12 December 2012
} 


\section{EXPERIMENTAL PROCEDURE}

\section{Materials}

1,4,5,8-Naphthalenetetracarboxylic dianhydride (NTDA, TCI, Tokyo, Japan) was dried in a vacuum. 4,4-Diamino-2,2-bipheyldislufonic acid (DAPS, TCI) was purified according to a reported method. ${ }^{20}$ Triethylamine (Wako Pure Chemicals, Tokyo, Japan) was distilled from $\mathrm{CaH}_{2} \cdot m$-Cresol (Wako Pure Chemicals) was distilled under reduced pressure. Benzoic acid (TCI), dimethylsulfoxide (Wako Pure Chemicals), 2,6-dihydroxynaphthalene (DHN, Wako Pure Chemicals) and other solvents and reagents were used as received.

\section{The synthesis of NDI-DAPS sulfonated polyimide}

The synthesis of SPI, whose chemical structure is shown in Scheme 1, was conducted with a one-pot chemical imidization method, as described in the literature. ${ }^{21}$ DAPS $(0.50 \mathrm{~g}, 1.45 \mathrm{mmol})$, triethylamine $(0.33 \mathrm{~g}, 3.30 \mathrm{mmol})$ and $m$-cresol $(20 \mathrm{ml})$ were placed in a $300-\mathrm{ml}$ four-neck round-bottom flask equipped with a thermometer and an $\mathrm{N}_{2}$ inlet/outlet. The dispersion was stirred at $140^{\circ} \mathrm{C}$ for $3 \mathrm{~h}$ under $\mathrm{N}_{2}$ to produce a homogeneous solution. NTDA $(0.67 \mathrm{~g}, 3.34 \mathrm{mmol})$ was added to the solution, which was then stirred at $170{ }^{\circ} \mathrm{C}$ for $15 \mathrm{~h}$ and at $190^{\circ} \mathrm{C}$ for $3 \mathrm{~h}$ under $\mathrm{N}_{2}$. After being cooled to room temperature, the solution was poured into a mixture of concentrated $\mathrm{HCl}$ $(200 \mathrm{ml})$ and methanol $(500 \mathrm{ml})$ to yield a yellow precipitate that was washed with methanol several times and dried in a vacuum at $80^{\circ} \mathrm{C}$ for $48 \mathrm{~h}$ to produce $1.15 \mathrm{~g}(93 \%)$ of homo SPI.

\section{Membrane preparation}

A typical preparation procedure for SPI/DHN hybrid films was used as follows. The SPI was dissolved in DMSO (3wt $\%$ ) at $80^{\circ} \mathrm{C}$ to obtain a homogeneous solution. Various concentrations of DHN DMSO solutions were added to the SPI solution. The mixture was cast into glass dishes and then dried under reduced pressure at room temperature. Residual DMSO was removed by heating in a vacuum at $120^{\circ} \mathrm{C}$ for $24 \mathrm{~h}$.

\section{Measurements}

${ }^{1} \mathrm{H}$ NMR spectroscopic measurements were conducted at $25^{\circ} \mathrm{C}$ with a BRUKER AV-400 spectrometer $(400 \mathrm{MHz})$ (Bruker, Karlsruhe, Germany) and dimethyl sulfoxide- $\mathrm{d}_{6}\left(\right.$ DMSO- $\left.d_{6}\right)$. The number-average molecular weight $\left(M_{\mathrm{n}}\right)$ and the molecular weight distribution $\left(M_{\mathrm{w}} / M_{\mathrm{n}}\right)$ of polymers were determined using gel permeation chromatography in $N, N$-dimethylformamide containing $10 \mathrm{~mm} \mathrm{LiBr}$ at $40^{\circ} \mathrm{C}$ on a guard column (Tosoh TSK guard column Super AW-L, Tosoh, Yamaguchi, Japan) and on two columns (Tosoh TSK gel super AW 4000 and 5000) equipped with a JASCO instrument (JASCO, Tokyo, Japan), which was equipped with a JASCO 2031 plus refractive index detector and a JASCO 2070 plus UV/Vis detector .

The visible spectra of the SPI and SPI/DHN DMSO solutions or $50 \mathrm{vol} \%$ of the DMSO aqueous solutions were measured with a Lambda 35 (PerkinElmer, Inc. Waltham, MA, USA). The NDI unit concentration in the SPI was prepared to $10 \mathrm{~mm}$. DHN concentrations were prepared from 0 to $20 \mathrm{~mm}$ in a cuvette. The visible spectra of the SPI and SPI/DHN hybrid films were measured using a Shimadzu UV-3600 with MPC-2600 (Shimadzu, Kyoto, Japan). The thickness of the obtained films measured $\sim 10 \mu \mathrm{m}$. The thickness was controlled by the mixed amounts of the SPI and DHN. The films were cut to measure $30-\mathrm{mm}$ long and $10-\mathrm{mm}$ wide. The films were affixed to the spectrometer with a solid sample attachment. The obtained results were normalized according to the measured films' thickness.

Differential scanning calorimetry was performed with a SII DSC6220 (SII, Chiba, Japan) at a heating and cooling rate of $10^{\circ} \mathrm{C} \mathrm{min}^{-1}$ in $\mathrm{N}_{2}$ flow. The mechanical properties of the SPI and the SPI/DHN hybrid films were evaluated with a tensile test. The films were fabricated into ISO 37-4 specimens (dumbbell shape, $12 \times 2 \times 0.03 \mathrm{~mm}$ ). The tensile modulus, tensile strength and elongation at break were measured in a Shimadzu EZ graph equipped with a $50-\mathrm{N}$ load cell at an across-head speed of $0.5 \mathrm{~mm} \mathrm{~min}^{-1}$. The data presented here represent the mean of four independent tests. Grazing incidence wide angle X-ray diffraction (GIWAXD) measurements of the SPI and the SPI/DHN hybrid films were conducted at BL13XU at SPring-8 (Japan Synchrotron Radiation Research Institute, Hyogo, Japan) The beam wavelength, exposure time, incident angle and step were $1.0 \AA, 65 \mathrm{~ms}, 0.16^{\circ}$ and $0.033^{\circ}$, respectively. The SPI and SPI/DHN hybrid films were dried at $120^{\circ} \mathrm{C}$ for $2 \mathrm{~h}$ in vacuo. The water uptake experiment was conducted by immersing the SPI and the hybrid films (30-40 mg per sheet) in water at room temperature for $1 \mathrm{~h}$. After the films were removed from water, the water droplets on the films were wiped off, and the immersed films were quickly weighed. The water uptake was calculated from Eq. (1), where $W_{\mathrm{d}}$ and $W_{\mathrm{s}}$ represent the weights of the dry and corresponding water-swollen membranes, respectively. Water absorption experiments were conducted using the dried films and a humidity-controllable oven. The dried films were placed under different humidity conditions $(25,45$, 60 and $80 \% \mathrm{RH}$ ) at $30^{\circ} \mathrm{C}$ overnight. The films were quickly weighed after being removed quickly from the oven. The water uptake was expressed as the number of water molecules per sulfonic group $(\lambda)$ from Eq 2, where $n\left(\mathrm{H}_{2} \mathrm{O}\right)$ is the $\mathrm{H}_{2} \mathrm{O}$ mole number, which is the water uptake value by weight, and $\mathrm{n}\left(\mathrm{SO}_{3}{ }^{-}\right)$was estimated from the weight of the dry membranes.

$$
\begin{aligned}
& \text { Water Uptake }(\%)=\left(\frac{W_{\mathrm{s}}}{W_{\mathrm{d}}}-1\right) \times 100 \\
& \lambda=\frac{n\left(\mathrm{H}_{2} \mathrm{O}\right)}{n\left(\mathrm{SO}_{3}^{-}\right)}
\end{aligned}
$$

$\lambda$ is the number of water molecules per sulfonic group.

\section{RESULTS AND DISUCUSSION}

\section{Polymer synthesis and film preparation}

The SPI was synthesized using chemical imidization and measured with ${ }^{1} \mathrm{H}$ NMR. The SPI had a high molecular weight and a wide polydispersity index (Supplementary Information, Supplementary Table S1). In this paper, we used the same polymer a lot for each measurement to remove the effects of molecular weight and polydispersity.

The SPI/DHN hybrid films were prepared on a flat petri dish by solvent casting from DMSO solutions of SPI/DHN. As indicated by the visible spectra of the SPI and DHN mixture solution, the existence of water in the mixture solution helps form CT complexes of SPI and DHN compared with using DMSO exclusively. However, we prepared smoother and more uniform SPI/DHN hybrid films from the DMSO solution than from the DMSO and water mixture solution. For this reason, we used DMSO solutions for film preparation. The films obtained from the SPI/DHN mixtures were dark brown, whereas the SPI film without DHN was brown, which is typical for a polyimide (Figure 1). This difference indicates that the DHN and NDI unit in the SPI formed CT complexes in the hybrid films.
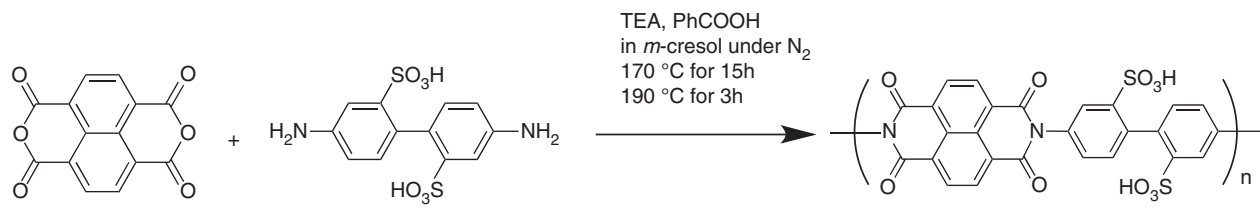

Scheme 1 The synthesis of sulfonated polyimide. 


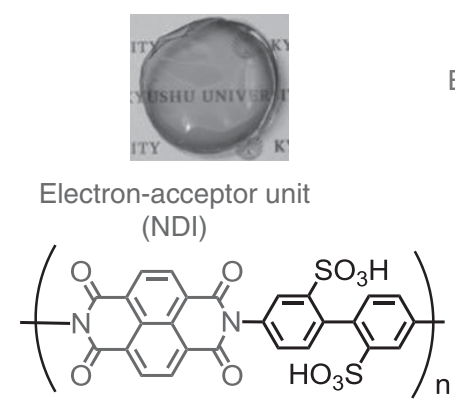

Sulfonated polyimide (SPI)

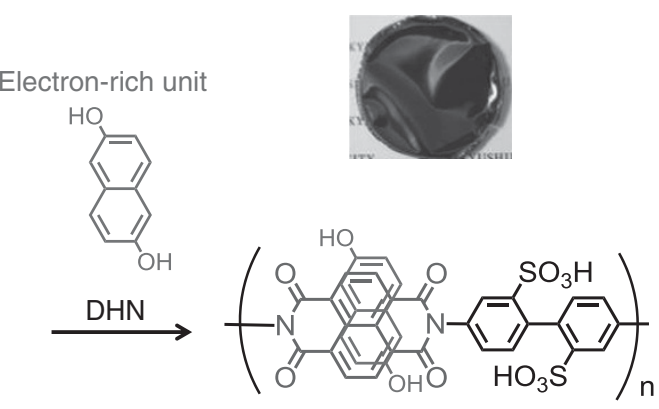

SPI/DHN hybrid
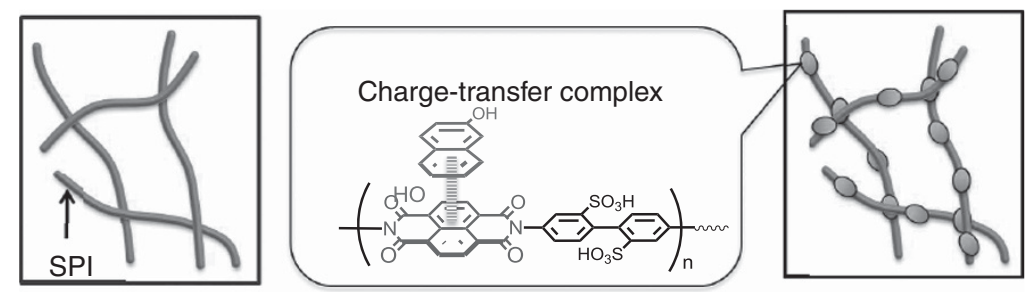

Figure 1 Schematic representation of the sulfonated polyimide/2,6-dihydroxynaphthalene (SPI/DHN) charge-transfer (CT) complex hybrid film and photographs of the SPI and SPI/DHN hybrid films. A full color version of this figure is available at Polymer Journal online.

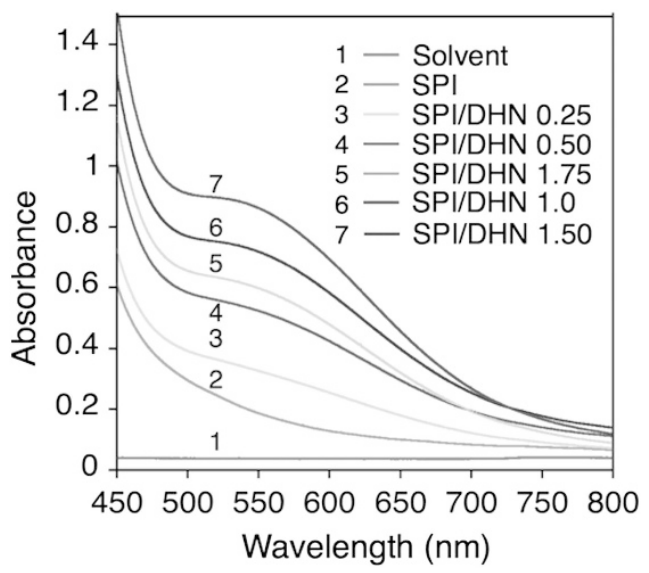

Figure 2 Visible absorption spectra of mixture solutions with sulfonated polyimide (SPI) and 2,6-dihydroxynaphthalene (DHN) in the DMSO/water mixture solution (SPI: $(\mathrm{NDI})=10 \mathrm{~mm})$. A full color version of this figure is available at Polymer Journal online.

\section{Visible spectroscopy (solution state and film state)}

To confirm the CT complex formation between NDI units in the SPI and DHN molecules, we measured the visible absorption spectra of various mixtures of SPI and DHN in solution. SPI/DHN solutions were prepared by adding DHN to the SPI DMSO solution. SPI/DHN is the molar ratio of the DHN to NDI units in the SPI. The SPI/DHN solution did not show peaks assignable to CT complexes, even at high concentrations of SPI/DHN $\left(2.5 \mathrm{mg} \mathrm{ml}^{-1}\right)$. In contrast, when we used a DMSO/water $(1 / 1, v / v)$ mixture, which can enhance interactions between the DHN and NDI units in the SPI, the SPI/DHN mixed solutions showed typical peaks $\left(\lambda_{\max }=530 \mathrm{~nm}\right)$ derived from CT complexes (Figure 2). As expected, an SPI solution without DHN did not show a strong peak from a CT complex, but weak CT complexes formed between SPIs. Moreover, DHN also did not show strong absorbance at $\sim 530 \mathrm{~nm}$. According to Iverson's results, the wavelength assigned to the CT complex in aqueous solution and the binding constant of NDI and DHN are $522 \mathrm{~nm}$ and $130 \mathrm{M}^{-1}$,

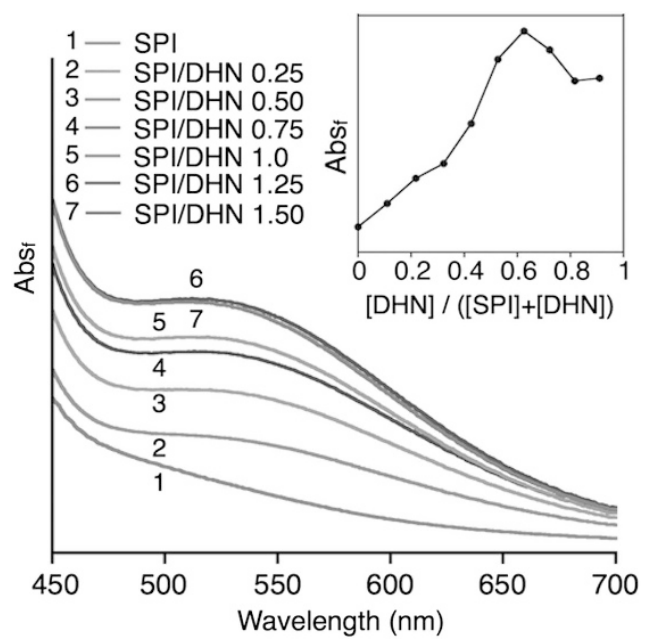

Figure 3 Visible absorption spectra of the hybrid films obtained from mixing the sulfonated polyimide (SPI) and 2,6-dihydroxynaphthalene (DHN) DMSO solution. Abs $f$ is the value normalized by the hybrid films' absorbance with respect to film thickness. The inset graphs the absorbance at $530 \mathrm{~nm}$ as a function of $[\mathrm{DHN}] /([\mathrm{SPI}]+[\mathrm{DHN}])$. A full color version of this figure is available at Polymer Journal online.

respectively. ${ }^{12}$ Our results are consistent with the previous results (the wavelength and binding constant were $530 \mathrm{~nm}$ and $172 \mathrm{M}^{-1}$, respectively). These results indicated that in the DMSO/water (1/1, $\mathrm{v} / \mathrm{v}$ ) solution, the formation of CT complexes between DHN and NDI units in the main chain of the SPI would be formed in preference to other CT complexes such as NDI-NDI and DHN-DHN complexes.

To quantitatively confirm the formation of CT complexes between NDI and DHN in the hybrid films, the visible spectra of the hybrid films were measured. These spectra showed an absorbance peak $\left(\lambda_{\max }=530 \mathrm{~nm}\right)$, which was absent in the spectrum of the SPI film without DHN (Figure 3). The results for the hybrid films correspond to those obtained from the solution sample. The peak at $\sim 530 \mathrm{~nm}$ represented a typical absorbance peak assigned to a CT band. 
Moreover, the CT absorbance between NDI and DHN increased with an increasing molar ratio of DHN to NDI units in the SPI and reached a maximum value when 1.3 equiv. of DHN to one NDI unit were used. Thus, we evaluated the stoichiometry of the CT complex formation between the DHN and NDI units in the SPI. The results obtained from a Job plot of the CT complex hybrid films showed that $>1$ equiv. of DHN was required to achieve maximum CT absorbance (Figure 3, inset), thus indicating that some DHN molecules could not form a CT complex with NDI during film formation. A major reason for this behavior is the low efficiency of the CT complex formation because of the low binding constant. Free DHN molecules, which did not form CT complexes with NDI units, would be located in other regions such as an amorphous region between the SPIs in the hybrid films.

\section{Physicochemical properties and chemical structures}

Some DHN molecules might crystallize or localize in the hybrid films, as indicated by the films' visible spectra. To confirm that the SPI and DHN were mixed homogeneously, we performed differential scanning calorimetric measurements of the CT hybrid films. DHN showed a clear endothermic peak at $\sim 230^{\circ} \mathrm{C}$, corresponding to DHN's melting point (Figure 4). In contrast, the SPI and the SPI/DHN hybrid films showed no endothermic peaks at $\sim 230^{\circ} \mathrm{C}$, indicating that $\mathrm{DHN}$ molecules were homogeneously dispersed in the SPI matrix.

To obtain further insights into the structures of the SPI/DHN hybrid films, we conducted grazing-incidence wide-angle X-ray diffraction (GIWAXD, BL13XU, SPring-8) measurements. Figure 5 shows the out-of-plane results. The SPI film showed a peak at approximately $q=16 \mathrm{~nm}^{-1}$, derived from the SPI-stacked structure, whereas the hybrid film showed two peaks at 16 and $12 \mathrm{~nm}^{-1}$. The peak at approximately $q=12 \mathrm{~nm}^{-1}$ indicates that the hybrid film has not only an SPI-stacked structure but also that different stacked states derived from the CT complex formation between NDI and DHN.

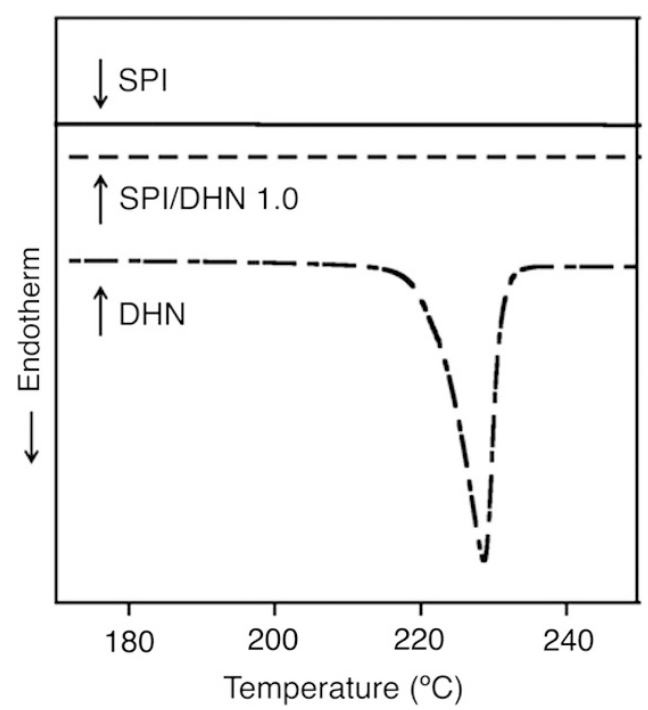

Figure 4 Differential scanning calorimetry (DSC) curves of SPI, sulfonated polyimide/2,6-dihydroxynaphthalene (SPI/DHN) 1.0 and DHN (heating process). The measured SPI and SPI/DHN were films, and the measured DHN was powder.

\section{Tensile test}

If DHN and NDI in the SPI main chain form a CT complex in the membrane, the CT complex should act as a specific binding site in the hybrid films. Cross-linking in the SPI/DHN hybrid films would generate a different mechanical strength from that of the original SPI film. Tensile tests were therefore conducted on the hybrid films. As shown in Figure 6, Young's modulus and stress at break increased with increasing amounts of DHN up to $[\mathrm{DHN}] /[\mathrm{SPI}]=0.8$ and reached $6 \mathrm{GPa}$ and $200 \mathrm{MPa}$, respectively. Young's modulus did not increase with subsequent additions of DHN, whereas the stress at break decreased with these additions. In contrast, the strain at break of the SPI/DHN hybrid films was $\sim 15 \%$ with additions of DHN up to $[\mathrm{DHN}] /[\mathrm{SPI}]=0.8$. This value was identical for the SPI film. When excessive DHN was added, the strain at break decreased to ca. $5 \%$. If DHN is mixed with the SPI matrix and disrupts the intermolecular interactions between the SPI molecular chains, the mechanical strength of the hybrid film should be lower than that of the SPI film. However, Young's modulus and the stress at break of the SPI/ DHN hybrid films increased up to $[\mathrm{DHN}] /[\mathrm{SPI}]=0.8$, thus indicating that the CT complex cross-links the SPI main chains through CT complex formation. However, the hybrid films containing excessive DHN became brittle compared with those formed using 0.8 equivalents of DHN. In other words, the mechanical properties degraded because the large amount of free DHN molecules in the hybrid films acting as mechanical defects affected the mechanical behavior. Normally, cross-linked materials become harder and more brittle than non-cross-linked materials do. However, the brittleness of the CT complex hybrid films and the SPI films did not differ notably in this system. CT interaction is weak interaction compared with other bonding such as hydrogen bonding and covalent bonding. In terms of weak interaction, CT complex in the film would not be able to form strong binding sites such as the cross-linkers formed by covalent bonding. Therefore, CT interaction would show a different mechanical property compared with the other cross-linked materials.

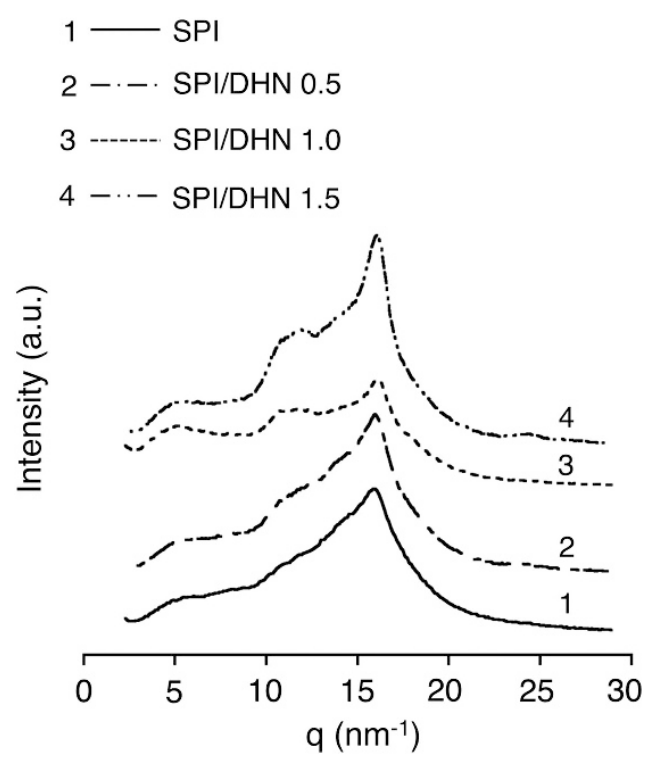

Figure 5 Grazing-incidence wide-angle X-ray diffraction (GIWAXD) profiles of the SPI and sulfonated polyimide/2,6-dihydroxynaphthalene (SPI/DHN) hybrid films (out-of-plane direction). 

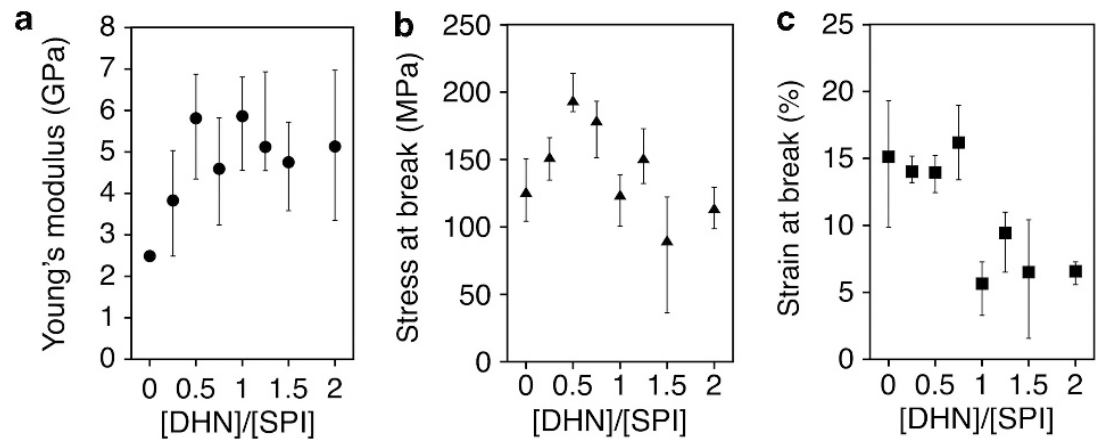

Figure 6 (a) Young's modulus, (b) stress at break and (c) strain at break of the hybrid films as a function of the ratio of molar equivalents of 2,6-dihydroxynaphthalene (DHN) units to 1,4,5,8-naphthalenediimide (NDI) units in the sulfonated polyimide (SPI).

Table 1 Water uptake and $\lambda$ values of SPI and SPI/DHN hybrid films

\begin{tabular}{lcc}
\hline Sample & Water uptake (\%) & $\lambda\left(\mathrm{H}_{2} \mathrm{O} / \mathrm{SO}_{3} \mathrm{H}\right)$ \\
\hline SPI & 160.7 & 26 \\
SPI/DHN0.5 & 120.8 & 22 \\
SPI/DHN1.0 & 45.3 & 9 \\
SPI/DHN1.5 & 56.4 & 13 \\
SPI/DHN2.0 & 32.0 & 8 \\
\hline
\end{tabular}

Abbreviations: SPI, sulfonated polyimide; DHN, 2,6-dihydroxynaphthalene.

\section{Water uptake}

SPIs are hydrophilic because of the presence of sulfonic acid groups. The CT complex formation between DHN and NDI in the SPI might affect the hybrid film's hydrophilicity. We therefore evaluated the water uptake of the SPI film and the SPI/DHN hybrid films. The water uptake of the SPI film immersed in water for $1 \mathrm{~h}$ at room temperature was $161 \%$ by weight (Table 1 ). The water uptake of the 0.5 and 1.0 equiv. hybrid films were $121 \%$ and $45 \%$, respectively. The number of water molecules per sulfonic acid in the SPI film, $\lambda$, was estimated to be 26 , whereas the $\lambda$ values of the hybrid films containing $>1$ equiv. of DHN were $<13$. DHN has two hydroxyl groups per molecule, and its solubility in water is approximately identical to that of the SPI (the solubility of DHN in water is $4.5 \mathrm{mg} \mathrm{ml}^{-1}$ at $50{ }^{\circ} \mathrm{C}$, and the solubility of the SPI in water is $5.0 \mathrm{mg} \mathrm{ml}^{-1}$ at $50^{\circ} \mathrm{C}$ ). Therefore, the decreased water uptake of the SPI/DHN hybrid films would be caused by suppressing swelling of the hybrid film by CT complex formation between NDI and DHN. This tendency was also observed in water adsorption of the films under constant humidity conditions. Under conditions of relative humidity $>45 \%$, the water adsorption of the SPI film was higher than that of the SPI/DHN hybrid films. This behavior also indicated that the CT complex formation between NDI in SPI and DHN would suppress water adsorption in the SPI/DHN hybrid films because of the enhancement of the structural stability by layered CT complex formation. When properties of the polyimides in water such as solubility and water uptake must be controlled, we consider the property of the monomer and do not control the property of the polyimides after polymerization. However, the film preparation method mentioned above can control the functions of the synthesized polyimides. The significant result of our film preparation method is the ability to conduct the post-modification of polyimides simply and easily.

\section{CONCLUSION}

In this paper, we demonstrated a versatile post-modification method for polyimides using CT complex formation between electrondeficient NDI and electron-rich DHN. Properties such as mechanical strength and water uptake were controllable, despite the simple filmpreparation method. The film preparation by CT complex formation reported in this paper is an attractive method for the functionalization of polyimides because a variety of donor molecules and polyimides can be used. The supramolecular methodology shown here is a simple but innovative method for polyimide modification.

\section{CONFLICT OF INTEREST}

The authors declare no conflict of interest.

\section{ACKNOWLEDGEMENTS}

The authors acknowledge partial financial support from the International Institute for Carbon-Neutral Energy Research (WPI- I2CNER), Kyushu University, Japan. The synchrotron radiation GIWAXD measurements were performed at the BL13XU (2011A1485) in the SPring-8 with the approval of the Japan Synchrotron Radiation Research Institute (JASRI).

1 You, N. H., Chueh, C. C., Liu, C. L., Ueda, M. \& Chen, W. C. Synthesis and memory device characteristics of new sulfur donor containing polyimides. Macromolecules 42, 4456-4463 (2009).

2 Tomioka, M., Suwa, M., Yoshida, S., Fujita, Y., Okuda, R. \& Ohbayashi, G. Novel positive-type photosensitive polyimide coatings "PW -1000". J. Photopolym. Sci. Technol. 13, 357-360 (2000).

3 Watson, K. A., Palmieri, F. L. \& Connell, J. W. Space environmentally stable polyimides and copolyimides derived from [2,4-bis(3-aminophenoxy)phenyl]diphenylphosphine oxide. Macromolecules 35, 4968-4974 (2002).

4 Ree, M. High performance polyimides for applications in microelectronics and flat panel displays. Macromol. Res. 14, 1-33 (2006).

5 Jang, W. Seo, J., Lee, C., Paek, S. H. \& Han, H. Residual stress and mechanical properties of polyimide thin films. J. Appl. Polym. Sci. 113, 976-983 (2009).

6 Sroog, C. E. Polyimides. Prog. Polym. Sci. 16, 561-694 (1991).

7 Hasegawa, T. \& Horie, K. Photophysics, photochemistry, and optical properties of polyimides. Prog. Polym. Sci. 26, 259-335 (2001).

8 Freilich, S. C. Photoconductivity of donor-loaded polyimides. Macromolecules 20, 973-978 (1987).

9 Chen, X., Chen, K., Chen, P., Higa, M., Okamoto, K.-I. \& Hirano, T. Effects of tetracarboxylic dianhydrides on the properties of sulfonated polyimides. J. Polym. Sci., Part A: Polym. Chem. 48, 905-915 (2010).

10 Yamazaki, K. \& Kawakami, H. High proton conductive and low gas permeable sulfonated graft copolyimide membrane. Macromolecules 43, 71857191 (2010).

11 Okamoto, K., Yaguchi, K., Yamamoto, H., Chen, K., Endo, N., Higa, M. \& Kita, H. Sulfonated polyimide hybrid membranes for polymer electrolyte fuel cell applications. J. Power Sources 195, 5856-5861 (2010). 
12 Lokey, R. S. \& Iverson, B. L. Synthetic molecules that fold into a pleated secondary structure in solution. Nature 375, 303-305 (1995).

13 Gabriel, G. J. \& Iverson, B. L. Aromatic oligomers that form hetero duplexes in aqueous solution. J. Am. Chem. Soc. 124, 15174-15175 (2002).

14 Bhosale, S., Sisson, A. L., Talukdar, P., Fürstenberg, A., Banerji, N., Vauthey, E., Bollot, G., Mareda, J., Röger, C., Würthner, F., Sakai, N. \& Matile, S. Photoproduction of proton gradients with pi-stacked fluorophore scaffolds in lipid bilayers. Science $\mathbf{3 1 3}$, 84-86 (2006).

15 Ramkumar, S. G. \& Ramakrishnan, S. Two-step folding of donor-acceptor foldamers. Macromolecules 43, 2307-2312 (2010).

16 Johnstone, K. D., Bampos, N., Sanders, J. K. M. \& Gunter, M. J. A self-assembling polymer-bound rotaxane under thermodynamic control. Chem. Commun. 12, 13961397 (2003).
17 Talukdar, P., Bollot, G., Mareda, J., Sakai, N. \& Matile, S. Synthetic ion channels with rigid-rod pi-stack architecture that open in response to charge-transfer complex formation. J. Am. Chem. Soc. 127, 6528-6529 (2005).

18 Cougnon, F. B. L., Au-Yeung, H. Y., Pantos, G. D. \& Sanders, J. K. M. Exploring the formation pathways of donor-acceptor catenanes in aqueous dynamic combinatorial libraries. J. Am. Chem. Soc. 133, 3198-3207 (2011).

19 Reczek, J. J. \& Iverson, B. L. Using aromatic donor acceptor interactions to affect macromolecular assembly. Macromolecules 39, 5601-5603 (2006).

20 Miyatake, K., Asano, N. \& Watanabe, M. Synthesis and properties of novel sulfonated polyimides containing 1,5-naphthylene moieties. J. Polym. Sci., Part A: Polym. Chem. 41, 3901-3907 (2003)

21 Kim, H., Litt, M. H., Nam, S. Y. \& Shin, E. Synthesis and characterization of sulfonated polyimide polymer electrolyte membranes. Macromol. Res. 11, 458-466 (2003).

Supplementary Information accompanies the paper on Polymer Journal website (http://www.nature.com/pj) 